\title{
La Situación de los Derechos Humanos de las Mujeres según el Comité para la Eliminación de la Discriminación Contra la Mujer \\ Observaciones Finales realizadas por el Comité al Cuarto Informe Periódico de Chile
}

Este comentario, junto a los documentos que analiza, está disponible en www.anuariocdh.uchile.cl

\author{
Cecilia Medina Quiroga*
}

\section{El examen de los Informes de Estados}

El Comité para la Eliminación de la Discriminación contra la Mujer (Comité CEDAW o Comité) es el órgano establecido por la Convención sobre Eliminación de Todas las Formas de Discriminación Contra la Mujer (CEDAW) ${ }^{1}$ para "examinar los progresos realizados en la aplicación de la Convención" por los Estados partes de ella. De conformidad con el artículo 18 de dicho instrumento, los Estados tienen la obligación de someter para examen del Comité "un informe sobre las medidas legislativas, judiciales, administrativas o de otra índole que hayan adoptado para hacer efectivas las disposiciones de la presente Convención y sobre los progresos realizados en este sentido". El Comité examina internamente este informe y prepara una lista de preguntas sobre temas que le presentan dudas; las que luego son contestadas por el Estado en forma escrita. Para la elaboración del listado, a menudo los miembros del Comité reciben información de organizaciones no gubernamentales ${ }^{2}$. Después de este intercambio de preguntas y respuestas el informe se analiza en una audiencia pública.

Chile presentó su cuarto Informe periódico en $2004^{3}$ y éste fue examinado por el Comité en sus sesiones 749 y 750, celebradas el 16 de agosto de 2006, emitiendo posteriormente sus Observaciones Finales con fecha 25 de agosto de $2006^{4}$. Asimismo, hubo un informe sombra ${ }^{5}$ substancial presentado por un conjunto de organizaciones no gubernamentales ${ }^{6}$. El Comité CEDAW envió a Chile una lista de preguntas ${ }^{7}$ que se respondieron por escrito ${ }^{8}$. El Informe de Chile fue

* Codirectora del Centro de Derechos Humanos, Facultad de Derecho, Universidad de Chile. Jueza de la Corte Interamericana de Derechos Humanos.

1 Adoptada por resolución 34/180, de 18 de diciembre de 1979; vigente desde el 3 de septiembre de 1981. Tiene, a noviembre de 2006, 185 Estados partes, que constituyen el 90\% de los Estados que componen las Naciones Unidas.

2 Esta información toma a menudo la forma de un "informe sombra", que contiene observaciones críticas de estas organizaciones al informe del Estado e información adicional sobre otros puntos que dicen relación con los derechos humanos que garantiza el tratado respectivo.

3 Comité CEDAW, "Examen de los informes presentados por los Estados partes con arreglo al artículo 18 de la Convención sobre la Eliminación de Todas las Formas de Discriminación Contra la Mujer-Cuarto informe periódico de los Estados partes- Chile", CEDAW/C/CHI/4.

4 Comité CEDAW, "Observaciones Finales del Comité para la Eliminación de la Discriminación contra la Mujer: Chile", documento CEDAW/C/CHI/CO/4.

5 Ver nota al pie $N^{\circ} 2$.

6 Centro de Estudios de la Mujer (CEM), Centro de Estudios para el Desarrollo de la Mujer (CEDEM); Comité de América Latina y del Caribe para la Defensa de los Derechos de la Mujer (CLADEM), Corporación de Desarrollo de la Mujer; La Morada; Corporación DOMOS; Corporación Humanas; Facultad Latinoamericana de Ciencias Sociales (FLACSO); Foro-Red de Salud y Derechos Sexuales y Reproductivos-Chile; Instituto de la Mujer, y Movimiento Pro Emancipación de la Mujer Chilena (MEMCH).

7 Comité CEDAW, "Lista de cuestiones y preguntas relativas al examen de los informes periódicos - Chile", $\mathrm{CEDAW} / \mathrm{C} / \mathrm{CHI} / \mathrm{Q} / 4$.

8 Comité CEDAW, "Respuestas a la lista de cuestiones y preguntas relativas al examen del cuarto informe periódico - Chile", CEDAW/C/CHI/Q/4/Add.1. 
voluminoso e incluyó una descripción detallada de la situación en el país con respecto a cada uno de los artículos de la Convención. Algunos de sus acápites mencionaron las dificultades políticas que el Gobierno tuvo y sigue teniendo para avanzar en algunas de las recomendaciones que el Comité CEDAW le hizo con motivo de los informes periódicos segundo y tercero, particularmente aquellas vinculadas con el área de los derechos sexuales y reproductivos.

El informe sombra elaborado por las organizaciones no gubernamentales ya mencionadas se desarrolló primordialmente sobre la base de las recomendaciones anteriores hechas por el Comité CEDAW y sobre la lista de preguntas enviadas y contestadas por Chile. Ello, sin dejar de lado un breve diagnóstico sobre la democracia en Chile, con el fin de vincularlo con la situación de las mujeres, señalando que existe hoy una profunda brecha entre una sociedad que cambia su estilo de vida vertiginosamente y un sistema político que no logra captar dichos cambios. Según este informe sombra la sociedad abandona paulatinamente la concepción tradicional de la familia y de los roles de hombres y mujeres para centrarse en los derechos de las personas. Sin embargo, el sistema político no ha sido capaz de incorporar esta perspectiva.

\section{Las Observaciones Finales}

El Comité, de manera similar a otras observaciones finales de los órganos de tratados, pone el acento no sólo en el ordenamiento jurídico chileno, sino que muy especialmente en la implementación de las leyes y en las prácticas administrativas o judiciales que pueden constituir obstáculos al objetivo de la Convención de combatir la discriminación contra las mujeres. También de manera similar a otros Estados, el informe de Chile contiene mucha información sobre legislación y menos en materia de aplicación de esa legislación por los órganos del Estado.

El Comité comienza por agradecer al Estado la presentación del informe y la respuesta que dio a sus preguntas específicas, así como el alto nivel de la delegación que compareció a presentarlo, presidida por la Ministra del Servicio Nacional de la Mujer. Mencionó como aspectos positivos en el cumplimiento de sus obligaciones internacionales: el nombramiento de un gabinete con un $50 \%$ de mujeres; la designación de un $48,4 \%$ de mujeres en puestos de alta jerarquía en la administración; la solidez del mecanismo institucional para el progreso de la situación de las mujeres; los esfuerzos para promover la igualdad de género y la incorporación de perspectivas de género en todas las políticas públicas; la aprobación y puesta en vigencia de varias leyes importantes, como las reformas a los Códigos Penal y Procesal Penal, la ley de los Tribunales de Familia, la de acoso sexual y la ley de Matrimonio Civil, todas las cuales introdujeron modificaciones o crearon normas nuevas para eliminar discriminaciones existentes ${ }^{9}$.

El grueso de las Observaciones, sin embargo, se centra en los principales motivos de preocupación del Comité y en las correspondientes recomendaciones para solucionar los incumplimientos al tratado que allí se mencionan. Por una parte, se enfatiza, en general, la necesidad de terminar con las disposiciones legales que discriminan contra las mujeres y de cubrir las lagunas legislativas que entrañan diferencias en el goce de sus derechos humanos. Asimismo, alienta al Estado a que se establezca un calendario claro de progreso y a que se aumente la concientización de los legisladores y de la sociedad en general sobre la urgencia de modificar las leyes discriminatorias y de adoptar medidas especiales para acelerar la igualdad de facto ${ }^{10}$. Por otra parte, el Comité se refiere a aspectos de la normativa chilena y de la práctica administrativa y judicial en lo relativo a la familia, la discriminación en el trabajo, la participación política y la asistencia en salud, con

9 Observaciones Finales, párrs. 2-7.

10 Ibídem, párr. 10. 
énfasis en la salud sexual y reproductiva, temas todos que se han tocado con mucha frecuencia en las observaciones que el Comité ha hecho al propio Chile y a otros Estados de la región y que revelan la similitud de problemas provenientes de una cultura hemisférica compartida.

En cuanto a las relaciones de familia, el Comité insiste en la necesidad de modificar las normas que permiten la existencia de un régimen patrimonial en el matrimonio que discrimina contra las mujeres (el régimen de sociedad conyugal) y la fijación de una edad mínima para contraer matrimonio (16 años según la última reforma) que no es compatible con el artículo 1 de la Convención sobre los Derechos del Niño, que pone el límite en 18 años $^{11}$.

Con respecto a la participación política, Chile había informado al Comité que en 1997 inició la tramitación de un proyecto de ley para incorporar el sistema de cuotas en las Leyes Orgánicas Constitucionales de los Partidos Políticos, Votaciones Populares y Escrutinios y de Municipalidades, pero que el proyecto no avanzó por la escasa importancia y prioridad dada por los partidos políticos, algunos de sus dirigentes, y otros personajes públicos. El Comité se preocupa por la lentitud de este proceso, ya que la participación de las mujeres en el Congreso y en los municipios sigue siendo escasa, e insta al Estado a adoptar medidas especiales para incrementarla. También, el Comité emplaza al Estado a tomar medidas para aumentar el número de mujeres en el servicio exterior $^{12}$.

En materia laboral, las preocupaciones del Comité se refieren a la significativa diferencia salarial entre hombres y mujeres, a pesar de que éstas tienen, en general, un mayor nivel de educación; a las altas tasas de desempleo de las mujeres, y a la desprotección legal de las trabajadoras de bajos ingresos, ya que sólo el 39,7\% de ellas tiene contrato de empleo con las consiguientes consecuencias que ello acarrea para el goce del sistema de seguridad social. Se recomienda al Estado que investigue las causas de esta situación, produzca estadísticas desagregadas por sexo y establezca un sistema general para supervisar los contratos de las mujeres trabajadoras temporales, tomando las medidas pertinentes para acabar con esta práctica discriminatoria ${ }^{13}$.

Un tema al que se le ha dado poca importancia en Chile, pero que preocupa al Comité por la severidad del sufrimiento de las personas que padecen por ello, es el de la trata de mujeres y la explotación de la prostitución. El Comité pide al Estado información acerca de las causas y el alcance de la trata en Chile como país de origen, de tránsito y destino, y le reprocha la falta de legislación nacional y la ausencia de medidas adecuadas para combatir estos fenómenos. Así, exhorta a Chile para que adopte una estrategia general y un plan de acción para lidiar con ellos ${ }^{14}$.

No es sorprendente que las mayores preocupaciones del Comité se adviertan en relación con la salud sexual y reproductiva y las consecuencias que ello trae para el goce de otros derechos humanos de las mujeres. Chile, como otros países de América Latina, tiene una tradición católica que no se inclina por los cambios en este campo de los derechos humanos de las mujeres. Constata este órgano las altas tasas de embarazos de adolescentes y la incidencia que esto tiene en el desarrollo de las niñas, en tanto causa principal del abandono escolar, y da una serie de recomendaciones destinadas a disminuir el problema. Aun cuando el Gobierno informa sobre algunos avances para promover la aplicación de la Ley 19.688, que consagra el derecho de las estudiantes embarazadas o madres lactantes a acceder a los establecimientos educacionales ${ }^{15}$,

\footnotetext{
11 Ibídem, párrs. 9 y 21.

12 Ibídem, párrs. 9, 13 y 14.

13 Ibídem, párrs. 11 y 12.

14 Ibídem, párrs. 15 y 16.

15 Respuestas a la lista de cuestiones, nota 7, párr. 12.
} 
el Comité requiere del Estado el fortalecimiento de las medidas tomadas para la continuación de la educación de las madres jóvenes y la supervisión de estas medidas para asegurar su cumplimiento. Recomienda, a su vez, el enjuiciamiento de los hombres mayores que mantienen relaciones sexuales con menores de edad y medidas educativas que favorezcan las uniones y la procreación responsables ${ }^{16}$.

Relacionado con lo anterior, el órgano que supervisa se refiere a su preocupación porque el aborto en cualquier circunstancia constituya un delito enjuiciable, que lleva a las mujeres a la búsqueda de abortos inseguros con obvios riesgos para su vida y su salud. Advierte, además, que el aborto clandestino es la causa principal de mortalidad materna ${ }^{17}$. Esta preocupación del Comité ya se había expresado en las Observaciones Finales hechas al segundo y tercer informes periódicos de Chile y se había vinculado con la norma legal que obliga a los médicos a denunciar a las mujeres que concurren al hospital cuando ha habido secuelas de un aborto clandestino. También el Comité contra la Tortura, al examinar el tercer informe periódico del Estado a dicho órgano había recomendado a Chile que "eliminara la práctica de extraer confesiones de enjuiciamiento de las mujeres que buscan atención médica de urgencia como resultado de abortos clandestinos e investigara y revisara las condenas dictadas en las causas en las que se hayan admitido como prueba las declaraciones obtenidas mediante coacción en estos casos" ${ }^{\prime 18}$. En este mismo sentido se había pronunciado, además, el Comité de Derechos Humanos al estudiar el cuarto informe periódico de Chile, recomendando medidas para exceptuar la penalización del aborto en ciertos casos y para proteger la confidencialidad entre médico y paciente ${ }^{19}$. Requerido Chile en este punto, la respuesta dada fue que no existía norma estableciendo el deber de los médicos de "extraer confesiones como parte del acto médico", pero que, como éste debía recoger información para un correcto diagnóstico, era posible que obtuviera conocimiento de situaciones de aborto inducido. En ese caso, se dijo, existe una colisión de normas entre la confidencialidad del acto médico (artículo 201 del Código Procesal Penal) y la obligación de todo funcionario público de denunciar la comisión de un delito, como es el aborto provocado (Artículo 84 del mismo Código). La respuesta sostuvo que en la práctica, generalmente, los profesionales de la salud optan por respetar la confidencialidad, pero que "en casos en que sus creencias religiosas son fuertes contra el aborto, deciden lo contrario y denuncian ante los Tribunales de Justicia" ${ }^{20}$.

En cuanto a la despenalización del aborto, el informe de Chile señala que no es propósito del Gobierno iniciar un proyecto de ley en este sentido, ni siquiera con relación al aborto terapéutico (que era permitido en Chile hasta 1989, cuando el régimen de Pinochet eliminó la norma), añadiendo que "la cerrada resistencia a analizar el fenómeno que afecta a decenas de miles de mujeres, por parte de medios de comunicación conservadores, sectores religiosos opuestos a toda referencia a dicha realidad, y partidos políticos que comparten las posiciones señaladas, han tenido un fuerte efecto en la opinión pública" ${ }^{21}$. En este mismo campo, el informe de Chile

16 Observaciones Finales, párrs. 17 y 18.

17 Ibídem, párr. 19.

18 CAT/C/CR/32/\%, párr. 7, citado en CEDAW, "Lista de cuestiones y preguntas", nota 5, párr. 9.

19 CCPR/C/79/ Add. 104, 30 de marzo de 1999, párr. 15.

20 Respuestas a la lista de cuestiones y preguntas, nota 7, párr. 9.

21 Cuarto Informe Periódico, párr. 285. Sin embargo, en los hechos, la opinión pública no parece ser contraria a la despenalización de toda forma de aborto. En la Segunda Encuesta Nacional de Opinión Pública realizada por la Universidad Diego Portales en 2006 (Radiografía Cultural: Familia y Valores) se señala que hay importantes niveles de acuerdo respecto de la legalización del aborto cuando la madre corre peligro -aborto terapéutico- $(65,1 \%)$ o el embarazo es producto de una violación (59,9\%). Estas cifras de acuerdo disminuyen frente a los abortos realizados por otras causales. Ver www.udp.cl. 
había señalado la dificultad de ratificar el Protocolo Facultativo de la CEDAW, explicando que el procedimiento para hacerlo exigía la aprobación del Congreso chileno y que allí se había encontrado un obstáculo mayor en su tramitación ante el Senado, aun después de haber sido aprobado por la Cámara de Diputados. La prensa escrita chilena había Ilevado a cabo una intensa campaña comunicacional en contra de la ratificación argumentando que ella implicaría una "entrega" de la soberanía nacional al Comité de CEDAW, que podría imponer en Chile una ley que permitiera el aborto. La más alta autoridad eclesiástica chilena compartió estos argumentos exponiéndolos en una Comisión del Senado y a ello se le dio también amplia publicidad en los medios de prensa. Como resultado de esta campaña, el Gobierno se habría visto obligado a retirar el proyecto de la tabla del Senado, el que está todavía pendiente ${ }^{22}$.

Sin perjuicio de la información proporcionada por el Gobierno de Chile sobre avances en la tarea de disminuir el embarazo en las adolescentes, tales como el trabajo que se realiza para ofrecer el acceso universal a la anticoncepción de emergencia ${ }^{23}$ y su establecimiento como objetivo de la década 2000-2010, el Comité recomienda que todos los tipos de anticoncepción segura y los métodos de planificación familiar estén ampliamente disponibles y asequibles, sin ninguna restricción; que se provea de servicios de maternidad segura y asistencia prenatal, y que se tomen las medidas necesarias para que las mujeres no tengan que recurrir a abortos ilegales como consecuencia de la falta de servicios adecuados para el control de la fecundidad ${ }^{24}$.

Finalmente, el Comité hace algunas recomendaciones generales al Estado. Por un lado, ampliar las consultas a las organizaciones no gubernamentales con respecto a la aplicación de la Convención, utilizando como base la Declaración y Plataforma de Acción de Beijing. Por otro lado, la difusión amplia de sus Observaciones Finales con el objeto de que la población, en particular los funcionarios de la administración pública, los políticos, los parlamentarios y las organizaciones de mujeres y de derechos humanos adquieran conciencia de las medidas adoptadas para asegurar la igualdad de facto y de jure de la mujer. El Comité enfatiza que la aplicación plena y efectiva de la Convención es indispensable para alcanzar los objetivos de desarrollo del Milenio y lo exhorta a integrar la perspectiva de género en todas sus actividades ${ }^{25}$.

\section{Conclusiones}

Estas Observaciones Finales ciertamente no tocan todos los puntos en los cuales el Estado chileno está en deuda con la Convención para la Eliminación de Todas las Formas de Discriminación contra la Mujer, sino solamente aquéllos que el Comité estima son los más importantes. Aun en

Por otro lado, en la Encuesta Nacional de Opinión: Percepciones de las Mujeres en torno a la despenalización del aborto, realizada en el año 2006 por la Corporación Humanas y el Departamento de Ciencia Política del Instituto de Asuntos Públicos de la Universidad de Chile, el 75\% de las entrevistadas (todas mayores de 18 años e inscritas en los Registros Electorales) expresó que debe despenalizarse el aborto terapéutico y el 69,1\% manifestó que debe hacerse con urgencia. A su vez, el 70,9 de las personas entrevistadas se mostró favorable a la despenalización cuando el embarazo es producto de una violación y el 68,4\% cuando el feto tiene una malformación grave. Ver: http://www.humanas.cl/publicaciones. asp.

22 Informe de Chile, nota 2, párrs. 92-94.

23 Un instructivo del Ministerio de Salud autorizó la entrega de la "píldora del día después" en los consultorios públicos a todas las mujeres mayores de 14 años, lo que provocó un intenso debate sobre la tensión entre la patria potestad de padres y madres y el rol estatal en la sexualidad de las adolescentes. Dicho instructivo fue impugnado judicialmente y, en diciembre de 2006, el Tribunal Constitucional de Chile declaró su inconstitucionalidad. Sin embargo, el Tribunal sólo se limitó a indicar que una disposición semejante debía establecerse mediante un Decreto Supremo y no mediante una resolución ministerial. No se pronunció sobre el fondo del asunto debatido.

24 Observaciones Finales, párr. 20.

25 Ibídem, párrs. 26-31. 
relación con éstos, Chile tiene todavía una gran tarea por delante, que no se facilita por la discrepancia entre miembros de los órganos estatales sobre el alcance y contenido de sus obligaciones internacionales en esta materia y sobre la necesidad de cumplir con ellas, tal como lo exige el tratado del cual Chile es parte. Creo que el progreso es una cuestión de tiempo y que, en la medida que el cambio cultural que se aprecia en Chile se haga más imperioso, las autoridades tendrán que adecuarse a él. 